\title{
Gyro Assisted Automatic Kill Switch for All Terrain Vehicle
}

\author{
Mohammad Raffi \\ Department of Automobile Engineering \\ Hindustan Institute of Technology \& Science \\ Chennai, Tamil Nadu
}

\author{
B. Niharika \\ Department of Electrical \& Electronics Engineering \\ Hindustan Institute of Technology \& Science \\ Chennai, Tamil Nadu
}

\begin{abstract}
There is always a risk of all-terrain vehicle catching fire after a rollover due to fuel leakage in case the engine's ignition is on. This could be prevented by the driver's using the engine kill switch, but sometimes the incident could be worst and driver may not have access to the kill switch or may be lying unconscious. This following scenario can be prevented using an automatic kill switch that can detect the vehicle roll over momentum, check whether the vehicle have or have not rested in upright straight position after a crash and kill the running engine if the vehicle has rested in tipped over position. This device consists of a Gyro Sensor which can measure the angles of elevation or roll and signal the driver about the maximum limitations of the vehicle that can take on terrain. The Gyro sensor senses the vehicle in its tipped over angle and using relay switch it cuts down either fuel supply to the engine or power supply to the spark plug depending on weather its petrol or diesel driven engine.
\end{abstract}

Keywords-Gyro Sensor, Relay Switch, Spark Flug, Fuel Line, Petrol Engine, Diesel Engine \& Kill Switch

\section{INTRODUCTION}

An emergency power shutdown (E-Stop) switch is also known as Kill Switch which can shut power off in an emergency which can also be termed as Emergency Power off $(E P O)$, is used in emergency situation which can shut down machinery, when it cannot be shut down in the usual manner. It uses a safety mechanism unlike a normal shut-down switch. This mechanism has tendency to shuts down all systems in order and turns off the machine without damage. Even if the equipment is damaged a kill switch is designed to abort the operation as quickly as possible and can be operated simply in order to quickly help a panicked operator with impaired executive functions or a by-stander can activate it.

The Gyro Assisted Kill switch is a device that is used to shut down the engine automatically, the purpose this switch is that we don't have to take our hand off the steering before shutting the engine, so we can avoid any accidental fire explosion due to fuel tank leakage. The Gyro Assisted Kill switch has used a Gyro Accelerometer sensor that can sense the angular rate sensors or angular velocity sensors of the vehicle in real time. This sensor is programed in such a way that it consists a threshold angular value with respect to vehicle traveling in elevated road that can under go either pitching or rolling moment. This threshold values are calculated with respect to the center of gravity position of the vehicle. Typically, every vehicle has a limitation in travelling over an elevated road and beyond the limitation the vehicle can roll either side ways or pitch in front or roll over backways. The gyro senses the angle of the vehicle while
ATV running over a terrain. it basically signals the driver when the vehicles elevation has reached to its maximum limitation and beyond which the chances of vehicle roll over is high. And if the vehicle has crashed or rolled off from the ground, the sensors checks the angle of the vehicle when it comes to rest after rolled over. If the angle is greater than the limited elevation angle of the vehicle, then the device cuts the power flowing to the spark plug of an engine through a relay for petrol engines or shuts of the fuel supply in diesel engine.

\section{METHODOLOGY}

A. Construction of the Device

In order to construct the device, the following equipment's have been used:

1. Gyro Accelerometer Sensor (Gyroscope)

2. MPU 6050 Module

3. Arduino UNO

4. PCB Board

5. Relay

The MPU 6050 is the core mother board of the device which consist embedded gyro accelerometer sensor that can sense motion of the vehicle with respect to its angular acceleration and angular velocity. The Arduino UNO consist of 6 analog inputs with $16 \mathrm{MHz}$ quartz crystal that can support MPU 6050 module. The program and the threshold values are completely stored in Arduino. The real time values sensed from MPU 6050 module is processed through the arduino depending on which the electromagnetic relay connected to arduino opens or closes the circuit. The whole circuit is connected and laid over a PCB board.

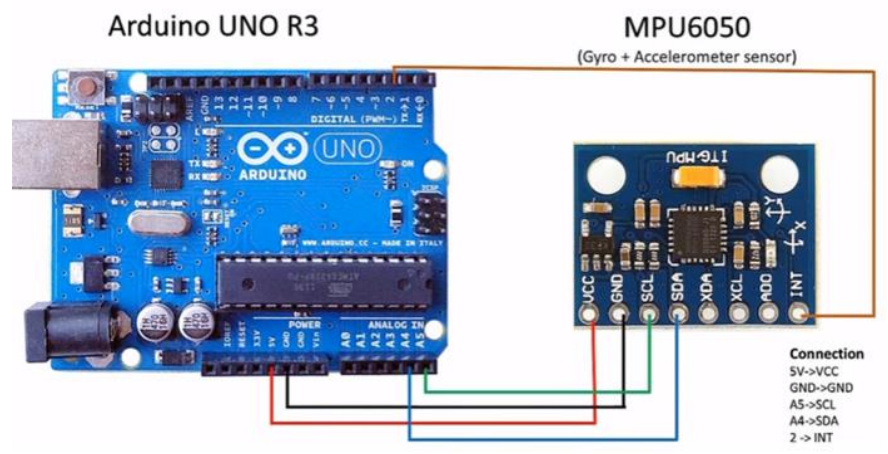

Fig. 1. Circuit Layout of the Device

\section{B. Working Procedure of the Device}

Initially the maximum angle of elevation of the vehicle with respect to its wheel track and wheel base is calculated considering center of gravity (CG) of the ATV. Then using 
simulation software, the maximum degree of CG shift in an banked road and the vehicle rolling angle at which the vehicle will roll over leaving the track is plotted in a graph subjecting to different velocities. Hence with respect to the graph manually in static the vehicle's balancing point and the maximum angle at which the vehicle will topple is found out using the device (Gyro Scope Embedded) fitted in vehicle.

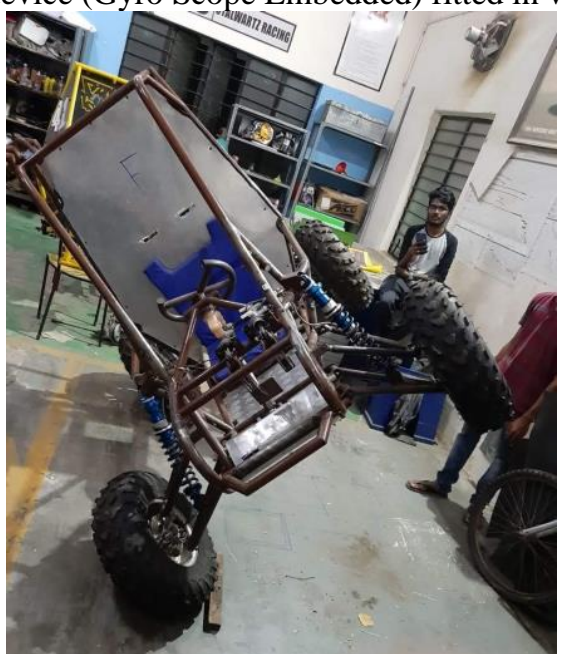

Fig. 2. ATV Balancing at its Center of Gravity with Maximum Angle of $40^{\circ}$

After finding out manually the maximum elevation limit of the ATV and threshold value is set to alert driver for safety \& caution. A time delay is set for the activation of kill switch with respect to the angle measured when the vehicle is tipped over.

Considering the following values, the below given steps are followed for calibration and installation of the device.

1. Initial step fix the device in vehicle mount, the gyro sensor activates once the vehicle battery turns $\mathrm{ON}$.

2. Once the vehicle run over the track, the gyro sensor continuously generates the angle of degree the vehicle is proceeding.

3. Arduino programming is done according to specification of vehicle needs with respect to the center of gravity of the vehicle, the tilt angle is set for warning indication.

4. Gyro sensor calibrate the angle at which vehicle is proceeding where when angle is greater than $40^{\circ}$ and less than $320^{\circ}$, a warning light is lightened up on driver dashboard.

5. If the calibrated angle is greater than $50^{\circ}$ and less than $310^{\circ}$ for more than 6 seconds, the relay switch is turned ON which functions to kill the engine.

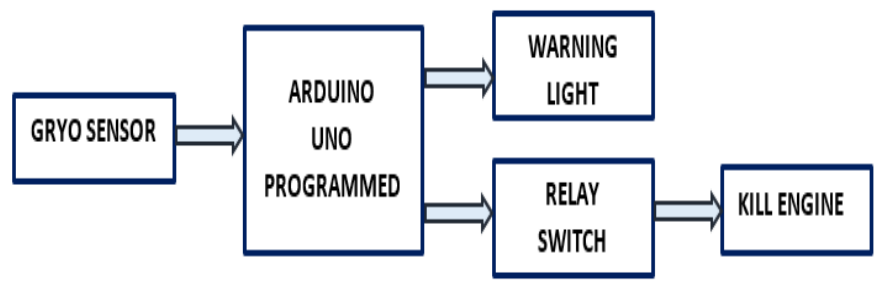

Fig. 3. Device Working Flow Chart

\section{PROGRAM CODE \& FLOW CHART}

\section{A. Program Codeing}

\#include<Wire.h>

const int MPU_addr $=0 \times 68$;

int16_t AcX,AcY,AcZ,Tmp,GyX,GyY,GyZ;

int $\min V a l=265$;

int $\operatorname{maxVal}=402$

int LED $=13$;

int RELAY $=12$;

double $\mathrm{x}$;

double y;

double $\mathrm{z}$;

void setup 0\{

Wire.begin();

Wire.beginTransmission(MPU_addr);

Wire.write(0x6B);

Wire.write(0);

Wire.endTransmission(true);

Serial.begin(9600);

pinMode(LED, OUTPUT);

\}

void loop 0\{

Wire.beginTransmission(MPU_addr);

Wire.write(0x3B);

Wire.endTransmission(false);

Wire.requestFrom(MPU_addr,14,true);

$\mathrm{AcX}=$ Wire.read $0<<8 \mid$ Wire.read 0 ;

AcY $=$ Wire.read $0<<8 \mid$ Wire.read 0 ;

$A c Z=$ Wire.read $0<<8 \mid$ Wire.read();

int $x$ Ang $=\operatorname{map}($ AcX, minVal, $\operatorname{maxVal},-90,90)$;

int $y$ Ang $=\operatorname{map}($ AcY, minVal, $m a x V a l,-90,90)$;

int zAng = map (AcZ, minVal, $\operatorname{maxVal},-90,90)$;

$\mathrm{x}=$ RAD_TO_DEG * (atan2(-yAng, -zAng)+PI);

$y=$ RAD_TO_DEG * (atan2(-xAng, $-\mathrm{zAng})+P I)$;

$\mathrm{z}=$ RAD_TO_DEG * (atan2(-yAng, $-\mathrm{xAng})+\mathrm{PI})$;

Serial.print("AngleX= ");

Serial.println(x);

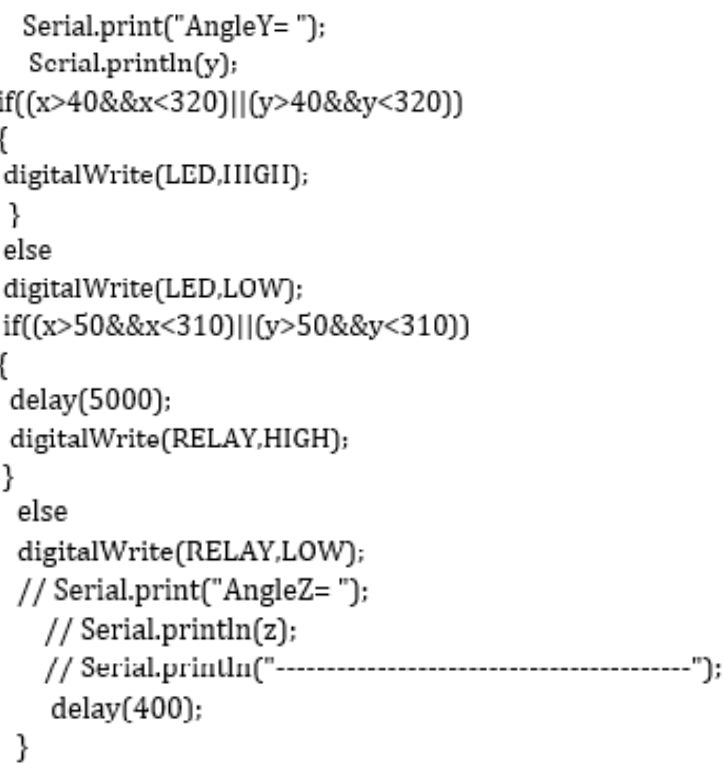


The following program is coded with the required values and stored in Arduino UNO.

\section{B. Flow Chart of the Program}

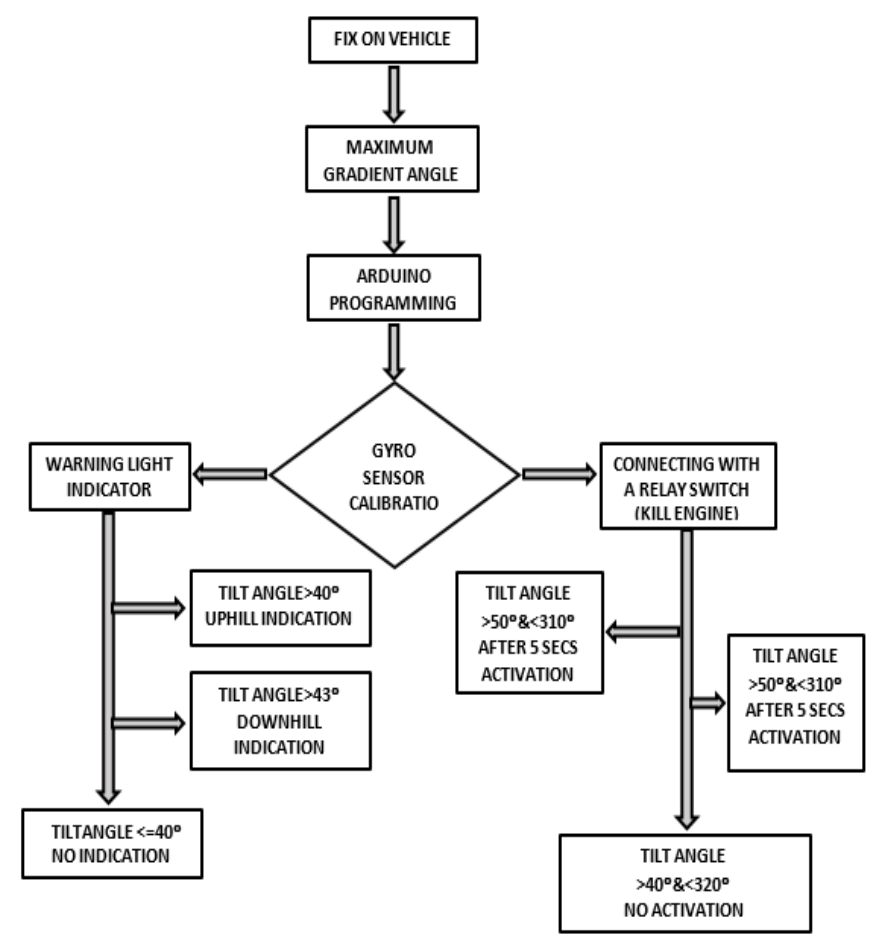

Fig. 4. Program Flow Chart

\section{CONCLUSION}

After when the device fitted in the petrol powered ATV, the relay switch was used to control the flow of current to the engine spark plug. When the ATV toppled, the relay created an open circuit to stop the flow of current from magneto induction coil of the engine to the spark plug. The relay circuit was activated after six seconds resulting in shutdown of engine.

During the case of normal running the driver was notified with light indication on the instrumental cluster whenever the driver reached the maximum rolling angle of the vehicle.

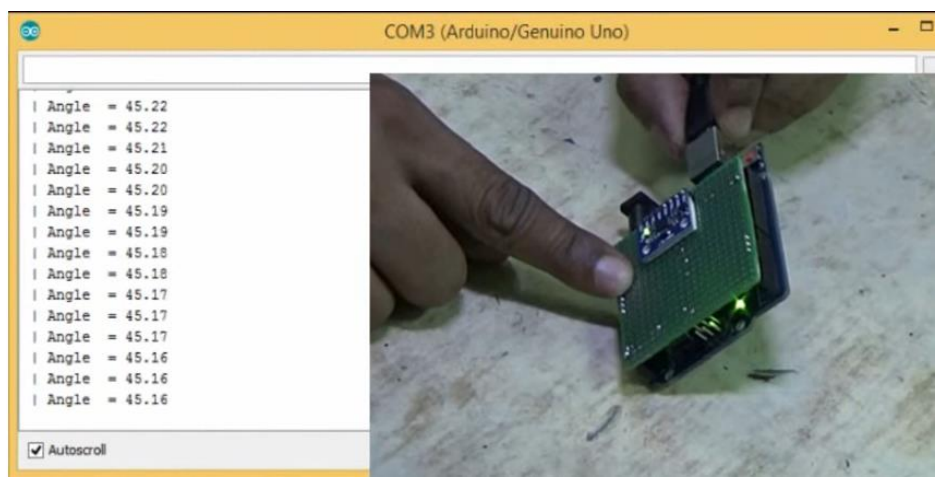

Fig. 5. Real-time ATV Elevation Angle Display.

\section{REFERENCES}

[1] Gillespie, Thomas d; "Fundamentals of vehicle dynamics", Warren dale, pa: society of automotive engineers Inc., year 1999

[2] Milliken, William f \& Milliken, Douglas 1; "Race car vehicle dynamics", warren dale, pa: society of automotive engineers Inc., year 1995

[3] Smith, Carroll; "Tune to win", Fallbrook, ca: aero publisher's Inc., year 1978

[4] G. Eason, B. Noble, and I.N. Sneddon, "On certain integrals of Lipschitz-Hankel type involving products of Bessel functions," Phil. Trans. Roy. Soc. London, vol. A247, pp. 529-551, April 1955. (references)

[5] J. Clerk Maxwell, A Treatise on Electricity and Magnetism, 3rd ed. vol. 2. Oxford: Clarendon, 1892, pp.68-73.

[6] I.S. Jacobs and C.P. Bean, "Fine particles, thin films and exchange anisotropy," in Magnetism, vol. III, G.T. Rado and H. Suhl, Eds. New York: Academic, 1963, pp. 271-350.

[7] Y. Yorozu, M. Hirano, K. Oka, and Y. Tagawa, "Electron spectroscopy studies on magneto-optical media and plastic substrate interface,” IEEE Transl. J. Magn. Japan, vol. 2, pp. 740-741, August 1987 [Digests 9th Annual Conf. Magnetics Japan, p. 301, 1982]. 